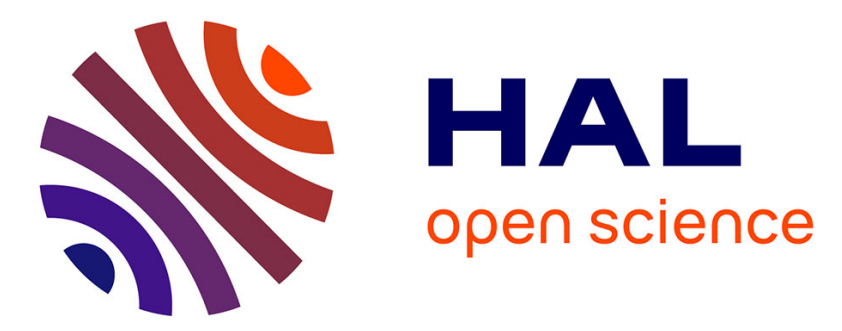

\title{
Pertinence clinique du calcul d'un score global à l'échelle de Conscience des Troubles Mentaux (SUMD)
}

\author{
C.M. Paillot, P. Ingrand, N. Jaafari
}

\section{To cite this version:}

C.M. Paillot, P. Ingrand, N. Jaafari. Pertinence clinique du calcul d'un score global à l'échelle de Conscience des Troubles Mentaux (SUMD). Annales Médico-Psychologiques, Revue Psychiatrique, 2011, 169 (7), pp.459. 10.1016/j.amp.2011.06.015 . hal-00789288

\section{HAL Id: hal-00789288 \\ https://hal.science/hal-00789288}

Submitted on 18 Feb 2013

HAL is a multi-disciplinary open access archive for the deposit and dissemination of scientific research documents, whether they are published or not. The documents may come from teaching and research institutions in France or abroad, or from public or private research centers.
L'archive ouverte pluridisciplinaire HAL, est destinée au dépôt et à la diffusion de documents scientifiques de niveau recherche, publiés ou non, émanant des établissements d'enseignement et de recherche français ou étrangers, des laboratoires publics ou privés. 


\section{Accepted Manuscript}

Title: Pertinence clinique du calcul d'un score global à

l'échelle de Conscience des Troubles Mentaux (SUMD)

Authors: C.M. Paillot, P. Ingrand, N. Jaafari

PII: $\quad$ S0003-4487(11)00174-0

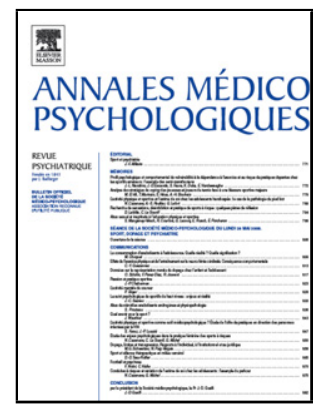

DOI: $\quad$ doi:10.1016/j.amp.2011.06.015

Reference: $\quad$ AMEPSY 1356

To appear in: $\quad$ Annales Médico-Psychologiques

Please cite this article as: Paillot CM, Ingrand P, Jaafari N, Pertinence clinique du calcul d'un score global à l'échelle de Conscience des Troubles Mentaux (SUMD), Annales medio-psychologiques (2010), doi:10.1016/j.amp.2011.06.015

This is a PDF file of an unedited manuscript that has been accepted for publication. As a service to our customers we are providing this early version of the manuscript. The manuscript will undergo copyediting, typesetting, and review of the resulting proof before it is published in its final form. Please note that during the production process errors may be discovered which could affect the content, and all legal disclaimers that apply to the journal pertain. 


\title{
Communication affichée
}

Influence de l'insight sur l'efficacité de l'entretien motivationnel dans la prévention des rechutes chez des patients alcoolo-dépendants

Insight and motivational interviewing effectiveness on relapse prevention in alcohol dependent patients

J. Lacoste ${ }^{1,3}$, M.L. Daniel ${ }^{1,3}$, F. Meissonnier ${ }^{2}$, M. Bacconnier ${ }^{1}$, J.L. Senon ${ }^{1}$, D. Belin ${ }^{3}$, N ; Jaafari ${ }^{1}$ pour Insight Study Group

1. Unité de Recherche Clinique en Psychiatrie, Centre Hospitalier Henri Laborit \& Inserm CIC-P 0802, CHU et Université de Poitiers, France

2. Centre Hospitalier Esquirol, Limoges, France

3. Inserm, Equipe AVENIR Psychobiologie des Désordres Compulsifs, IPBC - UMR 6187 CNRS et Université de Poitiers, France

adresse email :n.jaafari@wanadoo.fr

\section{Résumé}

Dans la prise en charge de l'alcoolo-dépendance, les interventions motivationnelles ont montré leur efficacité, notamment aux stades précoces du changement de Proschaska et Di Clemente. Chez 62 patients alcoolo-dépendants sans troubles cognitifs, nous montrons que l'efficacité de ces interventions dans la prévention de la rechute est liée au niveau d’insight, et que le bénéfice thérapeutique est significatif en cas de bon insight, mais est minime en cas de faible insight.

Mots clés : Alcoolo-dépendance ; Insight ; Prise en charge

\begin{abstract}
Motivational interventions are effective in the treatment of alcoholism, especially in the early stages of the Prochaska and DiClemente model of change. In 62 alcohol dependent patients without cognitive disorders, in addition to treatment as usual, motivational interviewing effectiveness on relapse prevention was linked with the level of insight. We have retrieved a moderate effectiveness on relapse prevention in patients with poor insight and a significant adjunctive effectiveness in patients with good insight.
\end{abstract}

Keywords: Alcohol dependence ; Insight

\section{Introduction}


L’alcoolo-dépendance est une maladie chronique, complexe et multifactorielle, avec des conséquences médicales, psychologiques et sociales importantes, qui concerne deux millions de personnes en France. Dans ses recommandations [1], la SFA préconise les interventions de type motivationnel dans l'accompagnement des patients alcoolo-dépendants, notamment aux stades précoces de changement de Proschaska et Di Clemente (pré-contemplation et contemplation). Cette psychothérapie, centrée sur l’information éclairée du patient, l'aide à la résolution de son ambivalence et la préparation au changement de son comportement, a pour objectif de mobiliser sa motivation, d'une manière totale ou partielle.

Cependant, ces approches pourraient être plus ou moins efficaces selon le niveau d'insight des patients.

\section{Objectif principal}

Notre objectif était d'évaluer l'impact de l'insight sur l'efficacité des entretiens motivationnels dans la prévention de la rechute chez des patients alcoolo-dépendants.

Critère d'évaluation principal : nombre de rechutes et de jours d'hospitalisation, trois et neuf mois après une hospitalisation pour sevrage.

\section{Méthodes}

Pendant six mois, tous les patients alcoolo-dépendants (critères du DSM-IV-TR) admis dans une unité d’hospitalisation du CH Esquirol de Limoges pour sevrage éthylique, après information détaillée et signature d’un consentement éclairé écrit, ont été inclus alternativement dans deux groupes, l’un bénéficiant de la prise en charge habituelle, l'autre d'entretiens motivationnels en association à la prise en charge habituelle.

La prise en charge habituelle comprend des entretiens réguliers avec un infirmier et le psychiatre référent de l'unité, combinés à un traitement médicamenteux adapté à chaque patient.

Les entretiens motivationnels ont été réalisés par des thérapeutes travaillant en structure d'alcoologie, et détachés pour les besoins de l'étude. Ils ont reçu une formation spécifique, pratiquent l'entretien motivationnel de façon courante, et sont intervenus exclusivement dans le cadre de ces entretiens.

\subsection{Critères d'inclusion}

Tout patient en difficulté avec l'alcool hospitalisé, avec demande de soins ou de sevrage, soit en motif d'hospitalisation principal, soit en motif d'hospitalisation secondaire (trouble de l'humeur associé, tentative d'autolyse, trouble du comportement, trouble psychotique chronique stabilisé...), quel que soit son mode d'hospitalisation (libre, d'office, à la demande d'un tiers). 


\subsection{Critères d'exclusion}

Patients avec détérioration cognitive sévère (syndrome de Korsakov, démence), avérée par la passation de tests cognitifs (Tests des 5 mots de Dubois inférieur à 8).

Patients avec troubles psychiatriques aigus ne permettant pas une bonne compliance aux soins (trouble psychotique aigu, manie, mélancolie).

Après deux à cinq jours de sevrage, un recueil des données sociodémographiques et une évaluation psychométrique (AUDIT, CDA, HDRS, HAMA) ont été réalisés, puis les patients ont été sollicités trois et neuf mois après leur hospitalisation et le nombre de rechutes et de jours d'hospitalisation ont été colligés pour chaque période. L’insight a été évalué par l’échelle Hanil Alcohol Insight Scale [2], qui a permis d'identifier des patients avec un insight bon ou faible.

\section{Résultats}

Soixante-deux patients alcoolo-dépendants sans troubles cognitifs ont participé à cette recherche. Les patients avec un bon insight semblent avoir une alcoolo-dépendance plus sévère (scores plus élevés à l'AUDIT - 27,26 vs 21,84, p<0,0001 - et consommation déclarée d'alcool plus importante - 325 vs $267 \mathrm{gr} / \mathrm{sem}, \mathrm{p}=0,029)$ que les patients avec un faible insight. Cependant, aucune différence significative n’a été retrouvée entre les deux groupes en termes d’âge de début $(\mathrm{p}=0,607)$ et de taux d'alcoolémie à l'admission ( $\mathrm{p}=0,578)$.

Par ailleurs, chez les patients ayant un bon insight, l'intervention motivationnelle diminue de manière significative le nombre de rechutes à trois et neuf mois $(p=0,04)$, alors que la diminution n'est pas significative $(p=0,45)$ chez les patients ayant un faible insight.

\section{Discussion}

Ces résultats soutiennent l'idée que les patients avec un bon insight pourraient avoir une estimation plus juste de leur pathologie que ceux avec un faible insight. Par ailleurs, l'intervention motivationnelle semble être moins efficace dans la prévention de la rechute en cas de faible insight.

\section{Conclusion}

Chez les patients alcoolo-dépendants, l'efficacité de l'ajout d'entretiens motivationnels à une prise en charge habituelle dépend du niveau d'insight et, si le bénéfice thérapeutique est significatif en cas de bon insight (en diminuant le nombre de rechutes et de jours d'hospitalisation à trois et neuf mois de sevrage), il paraît minime en cas de faible insight. 


\section{Pour en savoir plus}

1 ANAES, Conférence de Consensus : Modalités de l'accompagnement du sujet alcoolo-dépendant après un sevrage. Alcoologie Addictologie 2001;23:109-388.

2 Kim JS, Kim GJ, Lee JM, Lee CS, Oh JK. Hanil Alcohol Insight Scale (HAIS): validation of an insightevaluation instrument for practical use in alcoholism. J Stud Alcohol 1998;59:52-5. 